\title{
Collective relaxation dynamics of small-world networks
}

\author{
Carsten Grabow, ${ }^{1,2}$ Stefan Grosskinsky, ${ }^{3}$ Jürgen Kurths, ${ }^{1,4,5}$ and Marc Timme ${ }^{2,6,7}$ \\ ${ }^{1}$ Research Domain on Transdisciplinary Concepts and Methods, Potsdam Institute for Climate Impact Research, \\ P.O. Box 6012 03, 14412 Potsdam, Germany \\ ${ }^{2}$ Network Dynamics, Max Planck Institute for Dynamics and Self-Organization (MPIDS), 37077 Göttingen, Germany \\ ${ }^{3}$ Mathematics Institute and Centre for Complexity Science, University of Warwick, Coventry CV4 7AL, United Kingdom \\ ${ }^{4}$ Department of Physics, Humboldt University of Berlin, Newtonstr. 15, 12489 Berlin, Germany \\ ${ }^{5}$ Institute for Complex Systems and Mathematical Biology, University of Aberdeen, Aberdeen AB24 3UE, United Kingdom \\ ${ }^{6}$ Institute for Nonlinear Dynamics, Faculty for Physics, Georg August University Göttingen, 37077 Göttingen, Germany \\ ${ }^{7}$ Bernstein Center for Computational Neuroscience Göttingen, 37077 Göttingen, Germany
}

(Received 29 March 2015; published 27 May 2015)

\begin{abstract}
Complex networks exhibit a wide range of collective dynamic phenomena, including synchronization, diffusion, relaxation, and coordination processes. Their asymptotic dynamics is generically characterized by the local Jacobian, graph Laplacian, or a similar linear operator. The structure of networks with regular, small-world, and random connectivities are reasonably well understood, but their collective dynamical properties remain largely unknown. Here we present a two-stage mean-field theory to derive analytic expressions for network spectra. A single formula covers the spectrum from regular via small-world to strongly randomized topologies in Watts-Strogatz networks, explaining the simultaneous dependencies on network size $N$, average degree $k$, and topological randomness $q$. We present simplified analytic predictions for the second-largest and smallest eigenvalue, and numerical checks confirm our theoretical predictions for zero, small, and moderate topological randomness $q$, including the entire small-world regime. For large $q$ of the order of one, we apply standard random matrix theory, thereby overarching the full range from regular to randomized network topologies. These results may contribute to our analytic and mechanistic understanding of collective relaxation phenomena of network dynamical systems.
\end{abstract}

DOI: 10.1103/PhysRevE.91.052815

PACS number(s): 89.75.Hc, 05.45.Xt, 87.19.1m

\section{INTRODUCTION}

The structural features of complex networks underlie their collective dynamics such as synchronization, diffusion, relaxation, and coordination processes [1,2]. Such processes occur in various fields, ranging from opinion formation in social networks [3] and consensus dynamics of agents [4] to synchronization in biological circuits [5,6] and oscillations in gene regulatory networks and neural circuits [7-9]. The asymptotic collective dynamics of all such processes is characterized by the local Jacobian, the graph Laplacian, or similar linear operators. In general, such linear operators directly connect the structure of an underlying network to its dynamics and thus its function (see, e.g., Refs. [10,11]). Therefore, a broad area of research is related to the study of properties of such operators, in particular to the study of their spectra [12-19].

Small-world models based on rewiring have received widespread attention both theoretically and in applications, as demonstrated, for instance, by the huge number of references pointing to the original theoretical work [20]. But for most of their features analytical predictions are not known to date ([21]; a mean field solution of its average path length constitutes a notable exception [22]). In particular, the spectrum of small-world Laplacians has been studied for several specific cases and numerically [23-27], yet a general derivation of reliable analytic predictions was missing.

Here we present a mean field theory [28] toward closing this gap. The article is organized as follows. In Sec. II we first review the relations between relaxation dynamics and the spectrum of the graph Laplacian. In Sec. III we present rewiring "on average", a new mean-field rewiring recently proposed in a brief report [28]. Based on this rewiring, we derive a single formula that approximates well the entire spectrum from regular to strongly randomized topologies. We then investigate the ordering of the mean-field eigenspectrum in Sec. IV. In Sec. V we quantify the accuracy of our predictions via systematic numerical checks for the extreme eigenvalues. For the topological randomness $q$ of the order of unity, standard random matrix theory is applied in Sec. VI. We close in Sec. VII with a summary and a discussion of further work.

\section{NETWORK RELAXATION DYNAMICS}

The relaxation dynamics toward equilibrium and related collective phenomena close to invariant sets or stationary distributions emerge across a wide variety of systems ubiquitous in natural and artificial systems [1,29].

\section{A. Generic linear relaxation}

Mathematically, the relaxation of network dynamics to a fixed point, a periodic orbit, or a similar stationary regime is generically characterized by equations of the form

$$
\frac{d x_{i}}{d t}=\sum_{j=1}^{N} J_{i j}\left(x_{j}-x_{i}\right) \quad \text { for } i \in\{1, \ldots, N\},
$$

where $x_{i}(t)$ quantifies the deviation at time $t$ from an invariant state, $N \in \mathbb{N}$ is the size of the network, and $J_{i j} \in \mathbb{R}$ quantifies 
the influence of unit $j$ onto unit $i$. In general, $x_{i}(t) \in \mathbb{R}^{d}$, we here take $d=1$ for simplicity of presentation.

The equivalent mathematical description,

$$
\frac{d x_{i}}{d t}=\sum_{j=1}^{N} \Lambda_{i j} x_{j}
$$

for $i \in\{1, \ldots, N\}$, with the Laplacian

$$
\Lambda_{i j}= \begin{cases}J_{i j} & \text { for } i \neq j \\ -\sum_{j=1}^{N} J_{i j} & \text { for } i=j,\end{cases}
$$

follows directly from the original dynamics Eq. (1).

The eigenvalues $\lambda_{n} \in \mathbb{C}$ and corresponding eigenvectors $v_{n}$ of such a Laplacian, satisfying

$$
\Lambda v_{n}=\lambda_{n} v_{n}
$$

for $n \in\{1, \ldots, N\}$, fully characterize the asymptotic (linear or linearized) dynamics. For instance, for stable dynamics, where all $x_{i}(t) \rightarrow 0$ for $t \rightarrow \infty$, the largest nonzero (principal) eigenvalue $\lambda_{*}$ dominates the long-term dynamics: if we have $x_{j}(0)=\sum_{n=1}^{N} a_{n} v_{n}$, the vector $x(t)=\left[x_{1}(t), \ldots, x_{N}(t)\right]^{\top}$ evolves as

$$
\begin{aligned}
x(t) & =\exp (\Lambda t) x(0) \\
& =\exp (\Lambda t) \sum_{n} a_{n} v_{n} \\
& =\sum_{n=1}^{N} a_{n} \exp \left(\lambda_{n} t\right) v_{n} .
\end{aligned}
$$

Due to stability we have $a_{n}=0$ whenever $\lambda_{n}=0$, and for long times this is dominated by

$$
x(t) \sim a_{*} \exp \left(\lambda_{*} t\right) v_{*},
$$

where $\lambda_{*}$ is the principal eigenvalue.

Note that Eq. (5) also reveals how exactly all eigenvalues contribute to relaxation (and how much relative to each other). Additional individual eigenvalues of interest are given by the one with smallest real part $\lambda_{-}$, because it bounds the real parts of the spectrum below and thereby determines the support of the spectrum, and also because it is involved in determining synchronizability conditions in coupled chaotic systems via the ratio $\lambda_{*} / \lambda_{-}$, see Ref. [30].

\section{B. Different example systems}

We briefly consider two very different paradigmatic example classes of systems and comment on a few others whose relaxation properties are characterized by equations of the same type as Eq. (2).

\section{Stochastic processes}

First, consider random walks on a graph, or equivalently, Markov chains defined by a weighted nonnegative graph whose nodes represent the $N$ states [31]. For such processes, the dynamics of the probability $p_{i}(t)$ to reside in state $i \in\{1, \ldots, N\}$ at time $t$ is given by

$$
\frac{d p_{i}}{d t}=\sum_{j=1}^{N}\left[T_{i j} p_{j}-T_{j i} p_{i}\right] \text { for } i \in\{1, \ldots, N\},
$$

where $T_{i j}$ defines the transition rate (probability per unit time) of the system-switching state from $j$ to $i$ given it resides in $i$. We assume the process to be ergodic. Identifying $p^{*}$ to be the unique stationary distribution $\Lambda_{i j}=T_{i j}$ for $i \neq j$ and $\Lambda_{i i}=-\sum_{j} T_{j i}$ and setting $x_{i}(t) \equiv p_{i}(t)-p_{i}^{*}$ exactly maps this process onto the generic form of Eq. (2) with $x_{i}(t) \rightarrow 0$ as $t \rightarrow \infty$ for all $i \in\{1, \ldots, N\}$.

\section{Coupled deterministic oscillators}

Second, consider the relaxation dynamics of weakly coupled limit cycle oscillators, generically modeled as phasecoupled oscillators

$$
\frac{d \theta_{i}}{d t}=\omega_{i}+\sum_{j} h_{i j}\left(\theta_{j}-\theta_{i}\right) \quad \text { for } i \in\{1, \ldots, N\},
$$

where $\theta_{i}(t)$ is the phase of unit $i$ at time $t, \omega_{i}$ is the local intrinsic frequency of oscillator $i$, and $h_{i j}($.$) defines$ a smooth coupling function from unit $j$ to $i[9,32,33]$. Phase-locking, where $\theta_{j}(t)-\theta_{i}(t)=\Delta_{j i}$ is constant in time, constitutes a generic collective state of such systems, see, e.g., Refs. [34-36]. A paradigmatic model is given by networks of Kuramoto oscillators coupled by simple sinusoidal functions, i.e., $h_{i j}(\theta)=\sin (\theta)[32,33,37]$.

In the most general setting, a matrix $J$ is defined by elements $J_{i j}=\partial h_{i j}(\theta) /\left.\partial \theta\right|_{\theta=\Delta_{j i}}$ that encode the network structure close to the phase-locked state. Under certain conditions on the $\omega_{i}$ and the $h_{i j}$, the system's dynamics exhibits a short transient dominated by nonlinear effects and thereafter exponentially relaxes to the phase-locked state. Linearizing close to such a state yields phase perturbations defined as

$$
\delta_{i}(t):=\theta_{i}(t)-\theta(t)
$$

which evolve according to

$$
\frac{d \delta_{i}}{d t}=\sum_{j} \Lambda_{i j} \delta_{j}(t) \quad \text { for } i \in\{1, \ldots, N\},
$$

with the graph Laplacian given by Eq. (3).

In a simple setting, we have $J_{i j}=1$ for an existing edge and $J_{i j}=0$ for no edge such that the local linear operator in Eq. (10) coincides with the graph Laplacian defined by its elements,

$$
\Lambda_{i j}=J_{i j}\left(1-\delta_{i j}\right)-k_{i} \delta_{i j}
$$

for $i, j \in\{1, \ldots, N\}$, where now $J_{i j}$ are the elements of the adjacency matrix, $k_{i}$ is the degree of node $i$ (replaced by the in-degree for directed networks), and $\delta_{i j}$ is the Kronecker- $\delta$. The asymptotic relaxation dynamics on such networks is thus characterized by this graph Laplacian $\Lambda$. Similarly, any dynamics near genuine fixed points, for instance in gene regulatory networks [38,39], is equally characterized by linearized dynamics stemming from local Jacobians. 


\section{Power grids, social networks, neural circuits,...}

Several other systems exhibit qualitatively the same dynamical relaxation. In fact, power grids are often characterized by second-order oscillatory systems [40] that principally relax to periodic phase-locked solutions (stationary operating states of the grid) very similarly to the phase oscillator systems discussed above [41]. In models of several social phenomena, e.g., of opinion formation of agents, the dynamics of consensus formation is equally akin to such locking dynamics, where the locked state would now be a homogeneous, fully synchronous one [42]. Last but not least, perturbations of the spatiotemporal collective dynamics of pulse-coupled systems such as neural circuits $[43,44]$ also relax according to Eq. (2).

\section{MEAN FIELD REWIRING AND SPECTRUM}

Diving into explaining the small-world model, we analyze and derive its approximate Laplacian based on a two-stage mean-field rewiring. We follow Ref. [28] and where appropriate take parts of the description presented there.

We consider an initial ring graph of $N$ nodes. Each node receives $k$ (being even) links from its $k / 2$ nearest neighbors on both sides. Then we introduce randomness in the network topology by rewiring.

To define Watts-Strogatz randomized networks, single instances of an ensemble of stochastically rewired networks are generated (Fig. 1, left panel). Following Ref. [20] for undirected networks, we first cut each edge with probability $q$. Afterwards the cut edges are rewired to nodes chosen

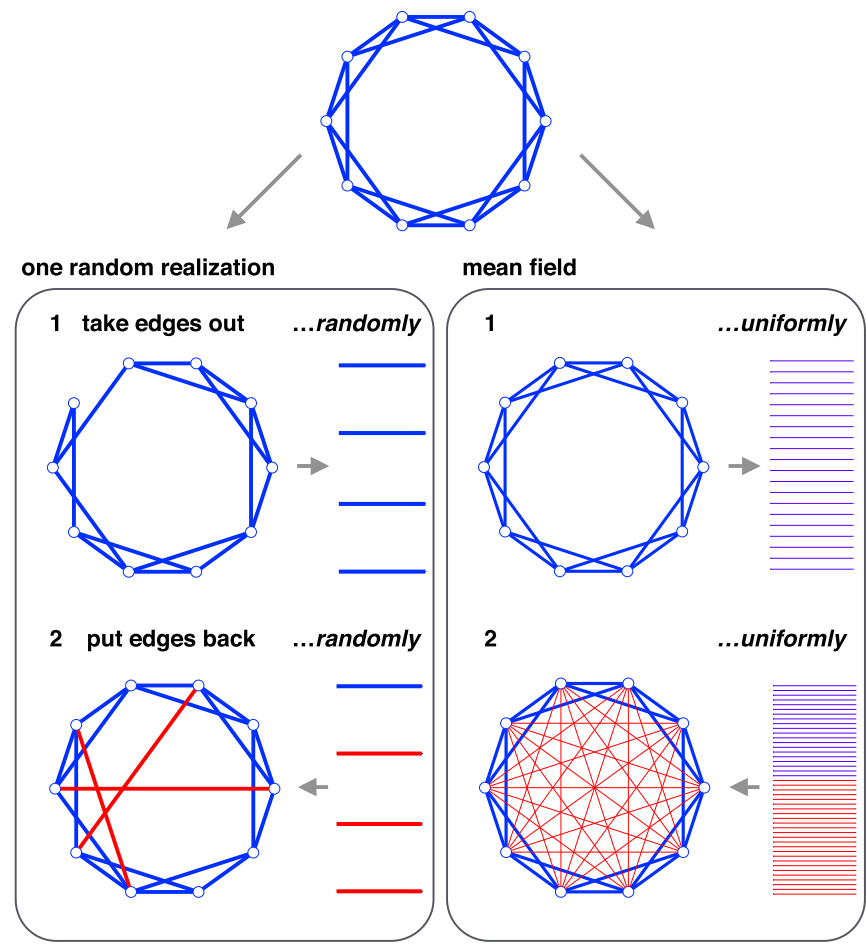

FIG. 1. (Color online) Rewiring-stochastic and mean field. Cartoon for $N=10$ and $k=4$. Instead of taking out (step 1) and putting back (step 2) edges randomly (left column), the corresponding weight is subtracted (step 1) uniformly and added (step 2) in two fractions (right column). uniformly at random from the whole network. Similarly, for directed [45] networks, we first cut all outgoing edges with probability $q$ and rewire their tips afterwards. In both cases we avoid double edges and self-loops.

To analytically determine the Laplacian mean-field spectrum in dependence of the network size $N$, the average degree $k$ and the topological randomness $q$, we introduce a two-stage mean-field rewiring that effectively generates, at given $q$, the average network from the ensemble of all stochastically rewired networks. This is depicted in Fig. 1 (right panel) in comparison to both other rewiring procedures for undirected and directed networks. First, we define a circulant mean-field Laplacian

$$
\tilde{\Lambda}^{\mathrm{mf}}=\left(\begin{array}{cccccc}
c_{0} & c_{1} & c_{2} & & \cdots & c_{N-1} \\
c_{N-1} & c_{0} & c_{1} & c_{2} & & \vdots \\
& c_{N-1} & c_{0} & c_{1} & \ddots & \\
\vdots & & \ddots & \ddots & \ddots & c_{2} \\
& & & & & c_{1} \\
c_{1} & \cdots & & & c_{N-1} & c_{0}
\end{array}\right) .
$$

Its matrix elements for the initial configuration (Fig. 1, $q=0$, top) are given by

$$
c_{i}= \begin{cases}-k & \text { if } i=0 \\ 1 & \text { if } i \in\left\{1, \ldots, \frac{k}{2}, N-\frac{k}{2}, \ldots, N-1\right\}=S_{1} \\ 0 & \text { if } i \in\left\{\frac{k}{2}+1, \ldots, N-\frac{k}{2}-1\right\}=S_{2},\end{cases}
$$

where $S_{1}$ represents the set of edges being present in the ring and $S_{2}$ those absent ones outside that ring.

Instead of rewiring single edges randomly (Fig. 1, left panel), we now distribute the corresponding weight of rewired edges uniformly among the whole network (Fig. 1, right panel). Thus, for a given rewiring probability $q$ we generate a mean-field version of the randomized network ensemble in the following two steps:

First, we subtract the average total weight $q k N / 2$ of all edges to be rewired $\left(S_{1}\right)$, i.e., $c_{i}=1-q$ if $i \in S_{1}$.

Second, the rewired weight is distributed uniformly among the total "available" weight in the whole network given by

$$
f=\frac{N(N-1)-(1-q) k N}{2} .
$$

With the weights

$$
f_{1}=\frac{q k N}{2}
$$

being available in $S_{1}$ and

$$
f_{2}=\frac{N(N-1)-k N}{2}
$$

in $S_{2}$, we assign the fraction $f_{1} / f$ to elements representing edges in $S_{1}$ and $f_{2} / f$ to those representing $S_{2}$. Therefore, an individual edge in $S_{1}$ gets the additional weight

$$
w_{1}=\frac{f_{1}}{f} \frac{\frac{q k N}{2}}{\frac{k N}{2}}=\frac{q^{2} k}{N-1-(1-q) k},
$$




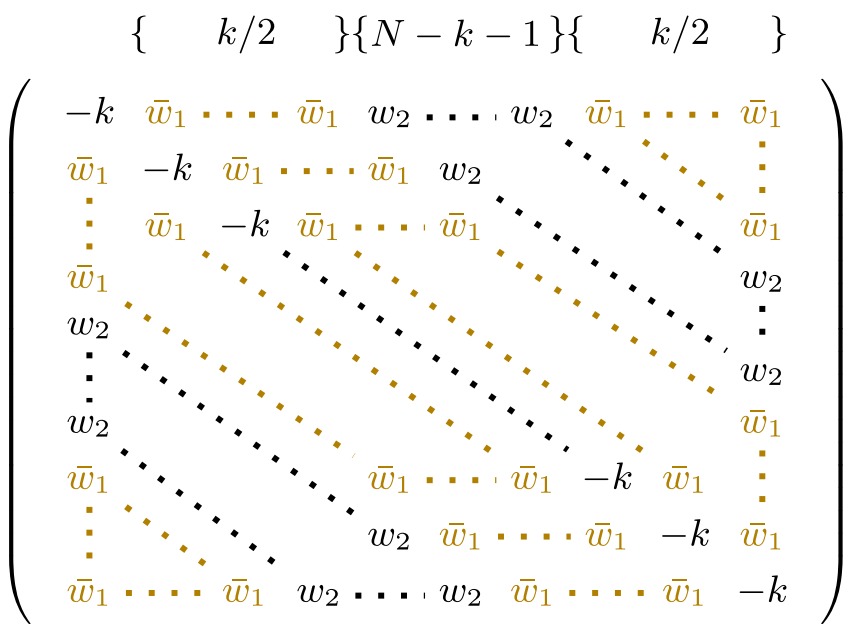

FIG. 2. (Color online) The banded structure of the mean-field graph Laplacian $\tilde{\Lambda}^{\mathrm{mf}}$ given in Eqs. (12) and (19). It has the weights $\bar{w}_{1}=1-q+w_{1}$ for $c_{i} \mid i \in S_{1}$ and $w_{2}$ for $c_{i} \mid i \in S_{2}$ [see Eq. (13) for the definition of $S_{i}$ ]. For $q=0$, and hence $\bar{w}_{1}=1$ and $w_{2}=0$, we recover the exact ring Laplacian.

and an edge in $S_{2}$ gets the new weight

$$
w_{2}=\frac{f_{2}}{f} \frac{\frac{q k N}{2}}{\frac{N(N-1)-k N}{2}}=\frac{q k}{N-1-(1-q) k} .
$$

Thus, as depicted in Fig. 2, in our mean-field theory the elements of the Laplacian $\tilde{\Lambda}^{\mathrm{mf}}$ Eq. (12) of a network on $N$ nodes with degree $k$ after rewiring with probability $q$ are given by

$$
c_{i}= \begin{cases}-k & \text { if } i=0 \\ 1-q+w_{1} & \text { if } i \in S_{1} \\ w_{2} & \text { if } i \in S_{2} .\end{cases}
$$

The mean-field Laplacian defined by Eqs. (12) and (19) by construction is a circulant matrix with eigenvalues [46-48]

$$
\tilde{\lambda}_{l}^{\mathrm{mf}}=\sum_{j=0}^{N-1} c_{j} \exp \left[\frac{-2 \pi i(l-1) j}{N}\right] .
$$

Observing the structure in Fig. 2 we immediately obtain the trivial eigenvalue for $l=1$ :

$\tilde{\lambda}_{1}^{\mathrm{mf}}=\sum_{j=0}^{N-1} c_{j}=-k+k\left(1-q+w_{1}\right)+(N-k-1) w_{2}=0$,

which is common to all networks (for all $q, N$, and any $k \leqslant N-1$ ) and reflects the invariance of Laplacian dynamics against uniform shifts, as seen from the associated eigenvector $\tilde{v}_{1}=(1, \ldots, 1)^{\top}$.

To obtain the remaining eigenvalues for $l \in\{2, \ldots, N\}$, we first define

$$
\begin{gathered}
x_{l}:=\exp \left[\frac{-2 \pi i(l-1)}{N}\right], \\
c^{\prime}:=\frac{1-q}{k}+q c^{\prime \prime},
\end{gathered}
$$

and

$$
c^{\prime \prime}:=\frac{q}{N-1-(1-q) k} .
$$

This leads to

$$
\begin{aligned}
\tilde{\lambda}_{l}^{\mathrm{mf}}= & -k+k c^{\prime} \sum_{j=1}^{\frac{k}{2}} x_{l}^{j}+k c^{\prime \prime} \sum_{j=\frac{k}{2}+1}^{N-1-\frac{k}{2}} x_{l}^{j}+k c^{\prime} \sum_{j=N-\frac{k}{2}}^{N-1} x_{l}^{j} \\
= & -k+k c^{\prime} \sum_{j=1}^{\frac{k}{2}} x_{l}^{j}+k c^{\prime} \sum_{j=1}^{\frac{k}{2}} x_{l}^{N-j} \\
& +k c^{\prime \prime}\left(\sum_{j=1}^{\frac{N-k}{2}-1} x_{l}^{\frac{N}{2}+j}+\sum_{j=1}^{\frac{N-k}{2}-1} x_{l}^{\frac{N}{2}-j}+x_{l}^{\frac{N}{2}}\right)
\end{aligned}
$$

where we have exploited the additional transposition symmetry $\tilde{\Lambda}^{\mathrm{mf}}=\left(\tilde{\Lambda}^{\mathrm{mf}}\right)^{\top}$, which implies $c_{j}=c_{N-j}$. Applying the Euler formula $\exp (i \alpha)=\cos (\alpha)+i \sin (\alpha)$, the complex summands cancel and we get

$$
\begin{aligned}
\tilde{\lambda}_{l}^{\mathrm{mf}}= & -k+2 k c^{\prime} \sum_{j=1}^{\frac{k}{2}} \cos \left[\frac{2 \pi(l-1) j}{N}\right] \\
& +x_{l}^{\frac{N}{2}} k c^{\prime \prime}\left\{2 \sum_{j=1}^{\frac{N-k}{2}-1} \cos \left[\frac{2 \pi(l-1) j}{N}\right]+1\right\} .
\end{aligned}
$$

Using the identity

$$
\begin{aligned}
\sum_{j=0}^{n} \cos (j \alpha) & =\cos \left(\frac{n+1}{2} \alpha\right) \sin \left(\frac{n \alpha}{2}\right) \frac{1}{\sin \left(\frac{\alpha}{2}\right)}+1 \\
& =\frac{1}{2}\left\{1+\frac{\sin \left[\left(n+\frac{1}{2}\right) \alpha\right]}{\sin \left(\frac{\alpha}{2}\right)}\right\},
\end{aligned}
$$

we obtain

$$
\begin{aligned}
\tilde{\lambda}_{l}^{\mathrm{mf}}= & -k+k c^{\prime}\left\{\frac{\sin \left[\frac{(k+1)(l-1) \pi}{N}\right]}{\sin \left[\frac{(l-1) \pi}{N}\right]}-1\right\} \\
& +x_{l}^{\frac{N}{2}} k c^{\prime \prime} \frac{\sin \left[\frac{(N-k-1)(l-1) \pi}{N}\right]}{\sin \left[\frac{(l-1) \pi}{N}\right]} .
\end{aligned}
$$

Taking advantage of additional identities, only valid for $l \in \mathbb{Z}$ (Fig. 3),

$$
\begin{aligned}
x_{l}^{N / 2} & =(-1)^{l-1}, \\
(-1)^{l-1} \sin (\alpha) & =\sin [\alpha+(l-1) \pi],
\end{aligned}
$$

and the symmetry $\sin (-\alpha)=-\sin (\alpha)$, the expression simplifies to

$$
\begin{aligned}
\tilde{\lambda}_{l}^{\mathrm{mf}}= & -k+k c^{\prime}\left\{\frac{\sin \left[\frac{(k+1)(l-1) \pi}{N}\right]}{\sin \left[\frac{(l-1) \pi}{N}\right]}-1\right\} \\
& +(-1)^{l-1} k c^{\prime \prime} \frac{\sin \left\{\frac{[-(k+1)+N](l-1) \pi}{N}\right\}}{\sin \left[\frac{(l-1) \pi}{N}\right]}
\end{aligned}
$$




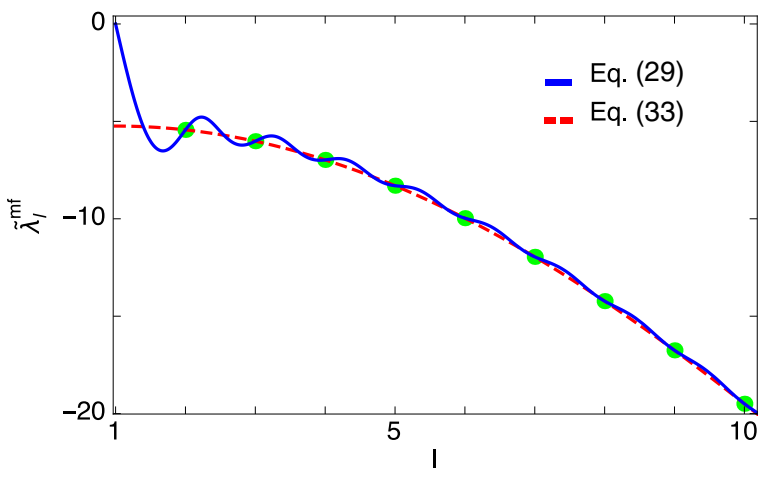

FIG. 3. (Color online) Interpolating the eigenvalues. Equations (29) (blue) and (33) (red) both contain the eigenspectrum $\tilde{\lambda}_{l}^{\mathrm{mf}}$ for $l \in\{2, \ldots, N\}$ correctly (green circles). While $\tilde{\lambda}_{l}^{\mathrm{mf}}$ in Eq. (29) includes $\tilde{\lambda}_{1}^{\mathrm{mf}}$ as $\lim _{l \rightarrow 1} \tilde{\lambda}_{l}^{\mathrm{mf}}=\tilde{\lambda}_{1}^{\mathrm{mf}}=0$ as well, $\tilde{\lambda}_{l}^{\mathrm{mf}}$ for $l \in\{2, \ldots, N\}$ in Eq. (33) does not: To further simplify expressions, we have used Eqs. (30) and (31) only valid for integer $l$, but apparently not for $l=0$.

$$
\begin{aligned}
= & -k+k c^{\prime}\left\{\frac{\sin \left[\frac{(k+1)(l-1) \pi}{N}\right]}{\sin \left[\frac{(l-1) \pi}{N}\right]}-1\right\} \\
& +k c^{\prime \prime} \frac{\sin \left\{\frac{[-(k+1)(l-1)+2 N(l-1)] \pi}{N}\right\}}{\sin \left[\frac{(l-1) \pi}{N}\right]} \\
= & -k+k c^{\prime}\left\{\frac{\sin \left[\frac{(k+1)(l-1) \pi}{N}\right]}{\sin \left[\frac{(l-1) \pi}{N}\right]}-1\right\} \\
& -k c^{\prime \prime} \frac{\sin \left[\frac{(k+1)(l-1) \pi}{N}\right]}{\sin \left[\frac{(l-1) \pi}{N}\right]},
\end{aligned}
$$

which finally leads to

$$
\tilde{\lambda}_{l}^{\mathrm{mf}}=-k-k c^{\prime}+k\left(c^{\prime}-c^{\prime \prime}\right) \frac{\sin \left[\frac{(k+1)(l-1) \pi}{N}\right]}{\sin \left[\frac{(l-1) \pi}{N}\right]},
$$

for $l \in\{2, \ldots, N\}$.

\section{THE ORDERING OF THE MEAN-FIELD SPECTRUM}

The spectrum obeys the symmetry

$$
\tilde{\lambda}_{l}^{\mathrm{mf}}=\tilde{\lambda}_{N-l+2}^{\mathrm{mf}},
$$

but is unordered otherwise; i.e., the index $l$ does neither denote eigenvalues with decreasing real part nor eigenvalues with decreasing absolute value.

As we argue below the expression $\tilde{\lambda}_{2}^{\mathrm{mf}}$ [which equals $\tilde{\lambda}_{N}^{\mathrm{mf}}$ due to Eq. (34)] always constitutes the second-largest (principal) eigenvalue $\tilde{\lambda}_{*}^{\mathrm{mf}}$. The only term depending on $l$ in Eq. (33) is the ratio

$$
\frac{\sin \left[\frac{(k+1)(l-1) \pi}{N}\right]}{\sin \left[\frac{(l-1) \pi}{N}\right]} .
$$

We therefore study the function

$$
f(x)=\frac{\sin [(k+1) x]}{\sin x},
$$

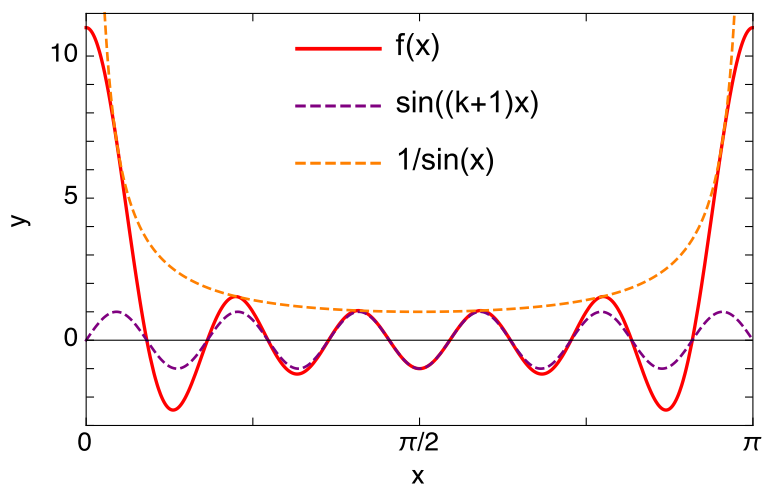

FIG. 4. (Color online) $\tilde{\lambda}_{2}^{\mathrm{mf}}$ always constitutes the second largest eigenvalue. Functions $f(x)$ [Eq. (36)], the oscillating function $\sin [(k+1) x]$, and the envelope function $1 / \sin (x)$ are plotted vs. $x=$ $\frac{(l-1) \pi}{N} \in(0, \pi)$ for $k=10$. Obviously, a larger $k$ leads to more roots of $f(x)$, but otherwise the functions show the same characteristics for all $k \leqslant N-1: f(x)$ has a local maximum at $x=0$ and decreases strictly monotonically up to the following minimum. For larger $x$ the envelope function guarantees that all values up to $x=\pi / 2$ are smaller than $f\left(x_{l=2}=\frac{\pi}{N}\right)$.

with

$$
x=\frac{(l-1) \pi}{N}
$$

and $x \in(0, \pi / 2)$. Due to the symmetry, Eq. (34), the interval $(0, \pi / 2)$ covers the entire spectrum, Eq. (33).

The function $f(x)$ on $x \in(0, \pi / 2)$ is the product of the oscillating function $\sin [(k+1) x]$ and a strictly monotonically decreasing function $1 / \sin (x)$. Therefore, it is a damped oscillation with period of $2 \pi /(k+1)$ and with the amplitude decreasing as $1 / \sin (x)$ (Fig. 4).

At $x=0$ we apply the Theorem of l'Hospital to calculate the following limits. There is a removable singularity,

$$
\lim _{x \rightarrow 0} f(x)=k+1,
$$

with

$$
\lim _{x \rightarrow 0} f^{\prime}(x)=0 \text { and } \lim _{x \rightarrow 0} f^{\prime \prime}(x)=-\frac{1}{3} k\left(k^{2}+3 k+2\right)<0,
$$

i.e., a local maximum.

In order to show that the index $l=2$ is always associated with the second-largest eigenvalue, we first determine its $x$ value. It is given by

$$
x_{l=2}=\frac{\pi}{N} .
$$

Since the roots of the function $f(x)$ are located at

$$
x_{\text {root }, r}=\frac{r \pi}{k+1},
$$

for $r \in \mathbb{Z}$. Thus, $x_{l=2}$ is always smaller than the first root $x_{\text {root }, 1}$, Eq. (41), of the function $f(x)$ (Fig. 5).

The boundary points of function $f(x)$ and the envelope function $1 / \sin (x)$ are given by

$$
x_{b, r}=\frac{4 \pi r+\pi}{2(k+1)},
$$

for $r \in \mathbb{Z}$. 


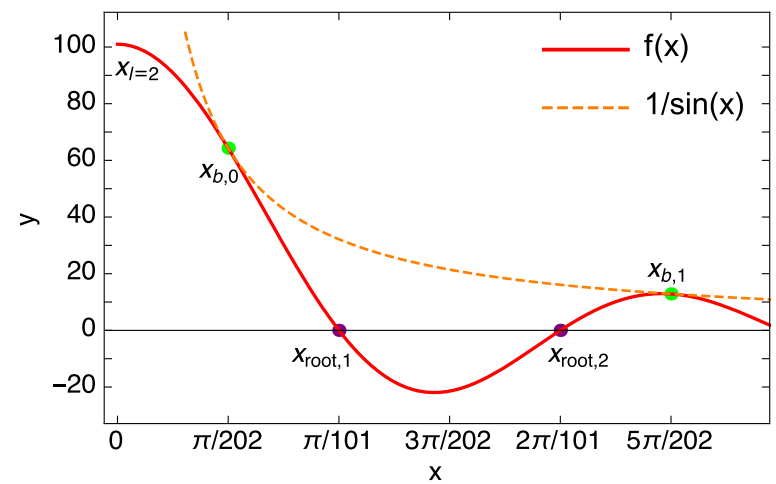

FIG. 5. (Color online) Important points of the function $f(x)$. The function $f(x)$ is plotted for $k=100$. The boundary points [green, Eq. (42)] of the function $f(x)$ and the envelope function $1 / \sin (x)$, roots of $f(x)$ [purple, Eq. (41)], and the $x$ value $x_{l=2}$ [blue, Eq. (40), $N=1000]$ corresponding to the eigenvalue $\tilde{\lambda}_{2}^{\mathrm{mf}}$ are highlighted.

The function $f(x)$ is bounded from above by the envelope function $1 / \sin (x)$ for all $x>x_{b, 0}$, with

$$
x_{b, 0}=\frac{\pi}{2(k+1)}
$$

being the first boundary point of function $f(x)$ and its envelope function $[r=0$ in Eq. (42)] (Fig. 5).

The first derivative of $f(x)$ stays negative at least up to the first root $[r=1$ in Eq. (41)] at

$$
x_{\text {root }, 1}=\frac{\pi}{k+1}>x_{b, 0},
$$

which is always larger than the first boundary point $x_{b, 0}$, Eq. (43).

To summarize, the function $f(x)$ has a local maximum at $x=0$ and is then strictly monotonically decreasing up to $x_{b, 0}$, Eq. (43). Then, for all $x>x_{b, 0}$ the function $f(x)$ takes values smaller than or at most equal to the values of the envelope function $1 / \sin (x)$, which is strictly monotonically decreasing in the considered domain (see Figs. 4 and 5). Thus, if $x_{l=2}$, Eq. (40), is smaller than the first boundary point $x_{b, 0}$, Eq. (43), the eigenvalue $\lambda_{2}$ constitutes indeed the second-largest eigenvalue.

Comparing Eqs. (40) and (43), this is the case for $N \geqslant$ $2(k+1)$, i.e., for $k<N / 2$. Numerical investigations suggest that the eigenvalue $\lambda_{2}$ always constitutes the second-largest eigenvalue independent from the chosen values for the parameters $N, k$, and $q$. However, monotonicity considerations are not that evident for $k>N / 2$.

The other extremal eigenvalue $\tilde{\lambda}_{-}^{\mathrm{mf}}$ cannot be that easily assigned to a fixed index. However, arguing similarly as for the second-largest eigenvalues, it is possible to find good estimates for the index at which the smallest eigenvalue,

$$
\tilde{\lambda}_{-}^{\mathrm{mf}}=\min _{l} \tilde{\lambda}_{l}^{\mathrm{mf}}
$$

always occurs.
The global minimum of $f(x)$ is always located between its first two roots $x_{\text {root, } 1}$ and $x_{\text {root, } 2}$ in Eq. (42); i.e.,

$$
x_{\text {root }, 1}=\frac{\pi}{k+1}<x_{-}<\frac{2 \pi}{k+1}=x_{\text {root }, 2} .
$$

It thus follows for the index $l_{-}$of the smallest eigenvalue:

$$
\frac{N}{k+1}<l_{-}-1<\frac{2 N}{k+1} \text {. }
$$

Therefore, a good estimate for the smallest eigenvalue is given by

$$
\tilde{\lambda}_{-}^{\mathrm{mf}} \approx \tilde{\lambda}_{\left.\frac{\mathrm{L} N}{2(k+1)}+1\right\rceil}^{\mathrm{mf}}
$$

where $\lfloor x\rceil$ denotes the nearest integer to $x$.

\section{EXTREME EIGENVALUES}

As the offset of each eigenvalue, Eq. (33), equals $k$, we consider the scaled eigenvalues

$$
\lambda_{l}^{\mathrm{mf}}(N, k, q)=\frac{\tilde{\lambda}_{l}^{\mathrm{mf}}(N, k, q)}{k}
$$

in the following to allow for a consistent analysis for different $k$. Additionally, we always plot the real part of the eigenvalues in the case of directed networks, whereas we plot $\lambda_{l} \in \mathbb{R}$ in the case of undirected networks.

As stated in the introduction, the principal eigenvalue $\lambda_{*}$ (that equals $\lambda_{2}$ as shown above) is of special importance since it dominates the long time dynamics (see, e.g., Ref. [49]).

For $l=2$, Eq. (33) simplifies to

$$
\begin{aligned}
\lambda_{2}^{\mathrm{mf}}(N, k, q)= & -1+c^{\prime}\left\{\frac{\sin \left[\frac{(k+1) \pi}{N}\right]}{\sin \left(\frac{\pi}{N}\right)}-1\right\} \\
& +c^{\prime \prime}\left\{\frac{\sin \left[\frac{(k+1) \pi}{N}\right]}{\sin \left(\frac{\pi}{N}\right)}\right\} .
\end{aligned}
$$

In Fig. 6 we compare the typical eigenvalues obtained by numerical diagonalization with our analytic prediction

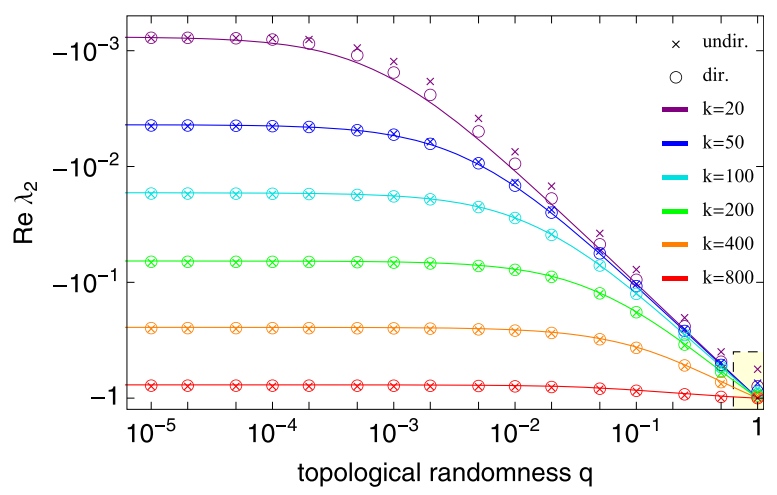

FIG. 6. (Color online) Second-largest eigenvalues from regular to randomized networks. Numerical measurements for undirected $(\times)$ and directed $(\bigcirc)$ networks in comparison with the analytical mean-field predictions [Eq. (50), solid lines] as a function of $q$, for different degrees $k$. The error bars on the numerical measurements are smaller than the data points $(N=1000$, each data point averaged over 100 realizations). Adapted from Ref. [28]. 


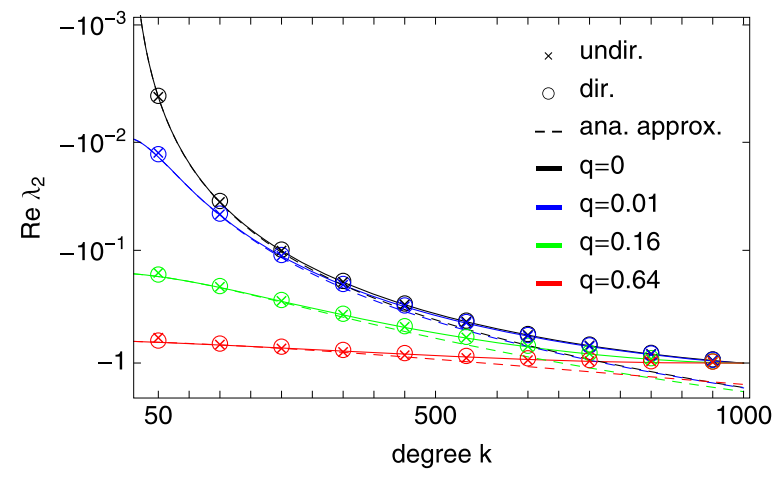

FIG. 7. (Color online) Scaling of the real part of the secondlargest eigenvalue for fixed network size. Numerical measurements for undirected $(x)$ and directed $(\bigcirc)$ networks in comparison with the analytical mean-field predictions [Eq. (50), solid lines]. The analytical approximations obtained from the expansions in Eq. (51) are depicted by the dashed lines. The error bars on the numerical measurements are smaller than the data points, $N$ fixed to 1000 .

Eq. (50). The analytic prediction is accurate for both undirected and directed networks, and for all but very small relative degrees $k / N<0.05$. Moreover, the prediction Eq. (50) approximates well the actual dependence of $\lambda_{2}$ for all but very large $q$, thus including regular-ring, smallworld, and even more substantially randomized network topologies.

Next we further investigate the scaling behavior of Eq. (50) in dependence on the system's parameters.

\section{A. Approximation for small degrees}

Expanding Eq. (50) up to $O\left(N^{-2}\right)$ as $N \rightarrow \infty$ yields

$$
\begin{aligned}
\lambda_{2}^{\mathrm{mf}}(N, k, q) \simeq & -q-\frac{[1+k(1-q)] q}{N} \\
& -\frac{(k+1)(k+2) \pi^{2}(1-q)+6 q(k+1-k q)^{2}}{6 N^{2}} .
\end{aligned}
$$

For $q=0$ we recover the known approximation for symmetric regular-ring networks:

$$
\lambda_{2}^{\mathrm{mf}}(N, k, 0) \simeq-\frac{(k+1)(k+2) \pi^{2}}{6 N^{2}} .
$$

The approximation Eq. (51) agrees well with Eq. (50) up to values of $k<N / 2$, but still is a good guide for even larger degrees $k$, cf. Fig. 7 .

\section{B. Scaling with network size}

In order to study the dependence on the network size we fix the edge density $d=k / N>0$ for large $N \gg 1$, which ensures that the network will remain connected. This leads to the expression

$$
\lambda_{2}^{\mathrm{mf}}(d, q) \simeq-1+\frac{(1-d)(1-q)}{[1-d(1-q)] d \pi} \sin (d \pi)
$$

in the limit $N \rightarrow \infty$.
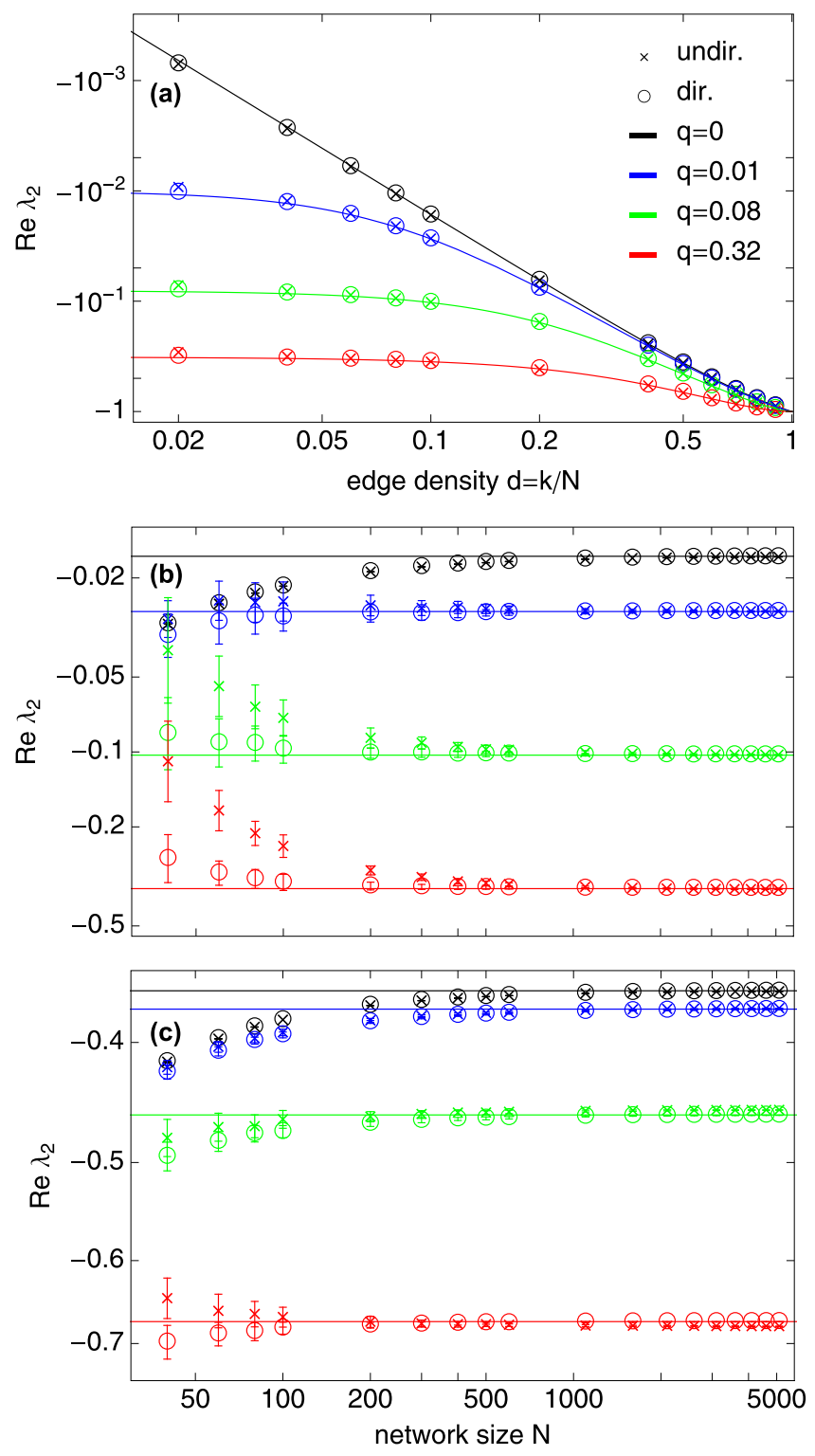

FIG. 8. (Color online) Second-largest eigenvalues in dependence on edge density and network size. (a) Numerical measurements for directed $(\bigcirc)$ and undirected $(\times)$ networks (error bars smaller than the data points) in comparison with the analytic mean field prediction (53) for $N=2000$. (b) Asymptotic $(N \rightarrow \infty)$ real parts of the secondlargest eigenvalues $\lambda_{2}$ in dependence on the network size $N$ for fixededge density $d=k / N=0.1$ [ $q$ values and symbols as in panel (a)]. (c) Asymptotic $(N \rightarrow \infty)$ real parts of the second-largest eigenvalues $\lambda_{2}$ in dependence on the network size $N$ for fixed-edge density $d=$ $k / N=0.5$ [ $q$ values and symbols as in (a)]. Panels (a) and (b) are adapted from Ref. [28].

Figure 8 confirms the validity of our approximation Eq. (53): the second-largest eigenvalues $\lambda_{2}$, for both undirected and directed networks, in dependence on the edge density $d$ for networks of size above about $N=500$ nodes are predicted well again. Edge densities other than those displayed $[d=k / N=0.1$ and $d=0.5$, Figs. 8(b) and 8(c)] qualitatively yield the same asymptotic behavior. 


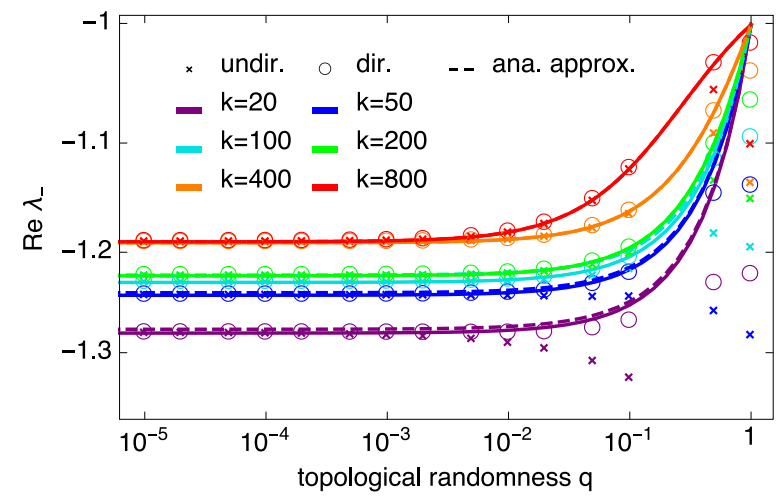

FIG. 9. (Color online) Smallest eigenvalues from regular to randomized networks. Numerical measurements for undirected $(x)$ and directed $(\bigcirc)$ networks in comparison with the analytical mean-field predictions [Eq. (45), solid lines] as a function of $q$, for different degrees $k$. Dashed lines show the analytic estimations of the smallest eigenvalues [Eq. (48)]. The error bars on the numerical measurements are smaller than the data points $(N=1000$, each data point averaged over 100 realizations).

\section{The smallest eigenvalue}

The smallest eigenvalue $\lambda_{-}$defined in Eq. (45) is also an important indicator for synchronization properties, in particular-in combination with the second-largest eigenvalue - for the synchronizability (see, e.g., Ref. [25]). For directed networks this refers to the eigenvalue with the smallest real part.

Here, the analytic prediction Eq. (33) again fits well with the actual eigenvalues obtained by numerical diagonalization, cf. Fig. 9. Note also that our estimation Eq. (48) for the smallest eigenvalue agrees well with the actual analytic prediction Eq. (45). It turns out that the analytic prediction is accurate for both undirected and directed networks. The prediction Eq. (50) approximates well the actual dependence of $\lambda_{-}$for small $q$, thus including regular rings and small worlds. The prediction is still a good guide for the general dependence of the second-largest eigenvalue on $q$, but shows some deviation from the numerical results for larger $q$, i.e., for substantially randomized network topologies.

\section{ANALYTICAL PREDICTIONS FOR RANDOM TOPOLOGIES ANALYTICAL PREDICTIONS VIA RANDOM MATRIX THEORY}

To analytically predict the second largest eigenvalues for the graph Laplacians of undirected and directed networks close to $q=1$ [see the shaded area (bottom, right) in Fig. 6] we consult random matrix theory [50] (cf. also Refs. [51-54]). For a review of synchronization in networks with random interactions, see, e.g., Ref. [55].

First, we consider undirected networks associated with symmetric matrices. Here, every connection between a pair of nodes $i$ and $j \neq i$ is present with a given probability $P$.

Second, we consider directed networks associated with asymmetric matrices. Here, all nodes have the same in-degree $k_{i}^{\text {in }}=k^{\text {in }}$. Each of the $k^{\text {in }}$ nodes that is connected to node $i$ is independently drawn from the set of all other nodes in the network with uniform probability.

Given a sufficiently large network size $N$ and a sufficiently large $k$ (respectively, a sufficiently large $k^{\text {in }}$ ), we numerically find that the set of nontrivial eigenvalues resemble disks of radii $r^{\prime}$ for undirected networks and $r$ for directed networks (cf. also Refs. [43,44]).

For directed networks where the in-degree $k_{i}^{\text {in }}=k$ for all nodes $i$ stays fixed during the whole rewiring procedure, i.e., all diagonal elements are constant, the graph Laplacian is obtained by shifting all eigenvalues of the adjacency matrix by $-k$. For undirected networks there are small deviations from node to node but the average degree equals $k$. However, numerical simulations confirm that shifting here again the eigenvalues of the symmetric adjacency matrix by the negative average degree $-k$ is feasible. Thus, we consider the adjacency matrices in the following, $A^{\text {sym }}$ for undirected and $A^{\text {asym }}$ for directed networks, and later shift them by $-k$.

\section{A. Ensembles of symmetric and asymmetric random matrices}

First, consider $N \times N$ symmetric matrices $A=A^{\top}$ with real elements $A_{i j}$. We constrain the diagonal entries to vanish $A_{i i}=0$ and denote its $N$ eigenvalues by $\lambda_{k}$. The elements $A_{i j}(i<j)$ are independent, identically distributed random variables according to a probability distribution $\rho\left(A_{i j}\right)$. According to Refs. [56-58], there is only one known ensemble with independent identically distributed matrix elements that differs from the Gaussian one. Thus, there are exactly two universality classes, i.e., classes that do not depend on the probability distribution $\rho\left(A_{i j}\right)$ but are determined by matrix symmetry only. Every ensemble of matrices within one of these universality classes exhibits the same distribution of eigenvalues in the limit of large matrices, $N \rightarrow \infty$, but the eigenvalue distributions are in general different for the two classes.

The arithmetic mean of the eigenvalues is zero,

$$
\left[\lambda_{i}\right]_{i}:=\frac{1}{N} \sum_{i=1}^{N} \lambda_{i}=\frac{1}{N} \sum_{i=1}^{N} A_{i i}=0,
$$

and the ensemble variance of the matrix elements scales like

$$
\sigma^{2}=\left\langle A_{i j}^{2}\right\rangle=\frac{r^{2}}{N}
$$

for $N \gg 1$ and $r>0$ being the radii of disks that enclose the set of nontrivial eigenvalues for directed networks $[43,44]$.

For the Gaussian symmetric ensemble, it is known [50,52] that the distribution of eigenvalues $\rho_{\text {Gauss }}^{\text {sym }}(\lambda)$ in the limit $N \rightarrow$ $\infty$ is given by Wigner's semicircle law:

$$
\rho_{\text {Gauss }}^{\text {sym }}(\lambda)= \begin{cases}\frac{1}{2 \pi r^{2}} \sqrt{4 r^{2}-\lambda^{2}} & \text { if }|\lambda| \leqslant 2 r \\ 0 & \text { otherwise. }\end{cases}
$$

The ensemble of sparse matrices [56,57,59-62] exhibits a different eigenvalue distribution $\rho_{\text {sparse }}^{\text {sym }}(\lambda)$ that depends on the finite number $k$ of nonzero entries per row and approaches the distribution $\rho_{\text {Gauss }}^{\text {sym }}(\lambda)$ in the limit of large $k$, such that

$$
\lim _{k \rightarrow \infty} \rho_{\text {sparse }}^{\text {sym }}(\lambda)=\rho_{\text {Gauss }}^{\text {sym }}(\lambda) .
$$


It is important to note that in the limit of large $N$ the eigenvalue distributions $\rho_{\text {sparse }}^{\text {sym }}$ and $\rho_{\text {Gauss }}^{\text {sym }}$ depend only on the one parameter $r$, which is derived from the variance of the matrix elements Eq. (55).

For real, asymmetric matrices (independent $A_{i j}$ and $A_{j i}$ ), there are no analytical results for the case of sparse matrices but only for the case of Gaussian random matrices. The Gaussian asymmetric ensemble yields the distribution of complex eigenvalues in a disk in the complex plane $[63,64]$

$$
\rho_{\text {Gauss }}^{\text {asym }}(\lambda)= \begin{cases}\frac{1}{\pi r^{2}} & \text { if }|\lambda| \leqslant r \\ 0 & \text { otherwise, }\end{cases}
$$

where $r$ from Eq. (55) is the radius of the disk that is centered around the origin. Like in the case of symmetric matrices, this distribution also depends only on one parameter $r$, which is derived from the variance of the matrix elements.

\section{B. Undirected random networks}

The real symmetric adjacency matrix $A^{\text {sym }}$ is an $N \times N$ matrix that satisfies $A_{i j}^{\mathrm{sym}}=A_{j i}^{\mathrm{sym}}$ and $A_{i i}^{\mathrm{sym}}=0$.

Furthermore, the matrix elements of $A^{\mathrm{sym}}$ are independent up to the symmetry constraint $A_{i j}^{\mathrm{sym}}=A_{j i}^{\mathrm{sym}}$. They are equal to 1 with probability

$$
P=\frac{\left\langle k_{i}\right\rangle}{N-1} \approx \frac{k}{N},
$$

and equal to 0 with probability $1-P$.

Thus, the variance $\sigma^{2}$ is given by

$$
\sigma^{2}=P(1-P)=\frac{k}{N}\left(1-\frac{k}{N}\right) .
$$

Therefore, the eigenvalues are located in a disk of radius

$$
r^{\prime}=2 r
$$

with

$$
r=\sigma \sqrt{N}=\sqrt{k-\frac{k^{2}}{N}}
$$

centered around the origin.

\section{Directed random networks}

The real asymmetric adjacency matrix $A^{\text {asym }}$ has exactly $k$ elements equal to one per row. Therefore, its elements have a spatial average

$$
\left[A_{i j}^{\text {asym }}\right]:=\frac{1}{N} \sum_{j=1}^{N} A_{i j}^{\text {asym }}=\frac{k}{N}
$$

and a second moment

$$
\left[\left(A_{i j}^{\mathrm{asym}}\right)^{2}\right]=\frac{1}{N} \sum_{j=1}^{N}\left(A_{i j}^{\mathrm{asym}}\right)^{2}=\frac{k}{N} .
$$

Thus, the variance

$$
\sigma^{2}=\left[\left(A_{i j}^{\mathrm{asym}}\right)^{2}\right]-\left[A_{i j}^{\mathrm{asym}}\right]^{2}=\frac{k}{N}-\frac{k^{2}}{N^{2}} .
$$

If we assume that the eigenvalue distribution for directed networks with fixed in-degree is similar to those for random matrices $[43,44]$, we obtain a prediction from Eq. (55), which yields

$$
r=\sigma \sqrt{N}=\sqrt{k-\frac{k^{2}}{N}}
$$

for the radius of the disk of eigenvalues centered around the origin.

\section{Predictions for the scaled graph Laplacians}

To obtain predictions for the eigenvalues of the appropriate graph Laplacian, we have to consider the shift by $-k$ (discussed in the beginning of this section) and the scaling factor $1 / k$ introduced in Eq. (49).

Together with Eq. (62), the second-largest eigenvalues for undirected networks close to $q=1$ [Fig. 10, (a)] are well predicted by Wigner's semicircle law (wsc):

$$
\begin{aligned}
\lambda_{2}^{\mathrm{wsc}}(N, k, 1) & =\frac{1}{k}\left(2 \sqrt{k-\frac{k^{2}}{N}}-k\right) \\
& =2 \sqrt{\frac{1}{k}-\frac{1}{N}}-1 .
\end{aligned}
$$

The real parts of the eigenvalues for directed networks close to $q=1$ [Fig. 10(b)] with Eq. (66) are with the theory of

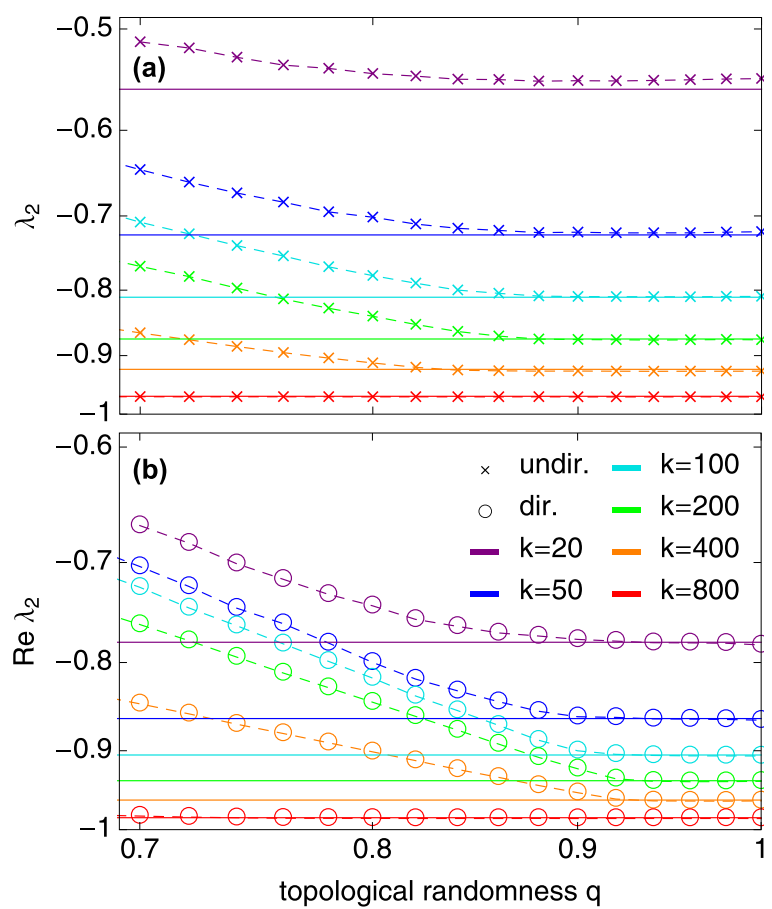

FIG. 10. (Color online) Analytic prediction of the second-largest eigenvalues close to $q=1$. (a) Numerical measurements for undirected $(\times)$ networks in comparison with the analytical predictions $\lambda_{2}^{\text {wsc }}$ via Wigner's semicircle law [Eq. (67), solid lines], for different degrees $k$. (b) Numerical measurements for directed $(\bigcirc)$ networks in comparison with the analytical predictions $\lambda_{2}^{\text {rmt }}$ from the theory of asymmetric random matrices [Eq. (68), solid lines]. The error bars on the numerical measurements are smaller than the data points ( $N=1000$, each data point averaged over 100 realizations). Dashed lines are only a guide to the eye. Taken from Ref. [28]. 
asymmetric random matrices (rmt),

$$
\begin{aligned}
\lambda_{2}^{\mathrm{rmt}}(N, k, 1) & =\frac{1}{k}\left(\sqrt{k-\frac{k^{2}}{N}}-k\right) \\
& =\sqrt{\frac{1}{k}-\frac{1}{N}}-1 .
\end{aligned}
$$

Note that $\lambda_{2}^{\text {wsc }}(N, k, 1)$ in Eq. (67) acquires a positive value for too small $k$ values and a sufficiently large network size $N$ (cf. [65]). However, for the $k$ values we investigated [Figs. 10(a) and 10(b)], the second-largest eigenvalues are well predicted by both Eqs. (67) and (68).

\section{SUMMARY AND DISCUSSION}

In this article we have presented and explicated derivations and extended a simple mean-field rewiring scheme suggested recently [28] to derive analytical predictions for the spectra of graph Laplacians. The key is replacing a stochastic realization of a rewired network at given topological randomness by its ensemble-averaged network. We achieve this averaging via a two-stage approach that distinguishes the original outer-ring structure and the originally "empty" inner part of a network and rewiring probabilities separately. For all $q$, the resulting average network in particular shares exactly the same (average) fraction of links in the original regular part of the network as well as in its originally "empty" part. We derive expressions for the largest nontrivial and the smallest eigenvalues, the full spectrum, as well as several scaling behaviors.

We remark that on theoretical grounds, the eigenvalue spectrum of the resulting average network in general is not equal to the average of the spectra of the individual stochastic network realizations. Yet, systematic numerical checks confirm that the mean-field approximation introduced is accurate as long as $q$ is sufficiently below one. In the limit $q \rightarrow 1$, we derive the eigenvalue spectra based on random matrix theory, which become exact in the limit of infinitely large networks, $N \rightarrow \infty$.
Although the mean-field rewiring is undirected, eigenvalues for directed networks are approximated more accurately and in a wider range of $q$ values, which is in particular related to the fact that the predictions for the undirected second-largest eigenvalues at $q=1$ are larger in real part than the directed ones, while all the mean-field eigenvalues converge to -1 at $q=1$. For "small" $k$ values the mean-field approximation becomes less accurate, which may be due to the fact that the ring structure is destroyed more easily while rewiring. Additionally, the bulk spectra spread much more drastically with $q$ than for larger $k$ values.

Furthermore, note that the analysis of the mean-field spectrum presented here can principally not be extended to the Laplacian eigenvectors as these are independent of the mean-field Laplacian's elements $c_{i}$ [19], just because of the circulant structure of the mean-field graph Laplacian. Consequently, the eigenvectors are the same and always nonlocalized, independent of the system's parameters $N, k$, and $q$. Studying distributed patterns of relaxation processes and potential localization phenomena thus requires access to eigenvectors beyond the mean-field approximation. Studies of the Laplacian eigenvectors are rare, although there are fascinating results as well. For instance, the discrete analogs of solutions of the Schrödinger equation on manifolds can be investigated on graphs (cf., e.g., Ref. [11]).

The simple mean-field approach presented above still substantially reduces computational efforts when studying randomized (regular or small-world) network models.

Generalizing our mean-field approach to higher dimensions and/or to other rewiring approaches, as for instance, relevant for neural network modeling [66], it will serve as a powerful tool to gain new insights into the relations between structural and dynamical properties of complex networks.

\section{ACKNOWLEDGMENTS}

This work was supported by the BMBF, Grants No. 03SF0472A (C.G., J.K.) and No. 03SF0472E (M.T.), by a grant of the Max Planck Society (M.T.), and by the Engineering and Physical Sciences Research Council (EPSRC) Grant No. EP/E501311/1 (S.G.).
[1] A. Pikovsky, M. Rosenblum, and J. Kurths, Synchronization, A Universal Concept in Nonlinear Sciences, Vol. 12 of Cambridge Nonlinear Science Series (Cambridge University Press, Cambridge, UK, 2001).

[2] S. Strogatz, Nature 410, 268 (2001).

[3] A. Pluchino, V. Latora, and A. Rapisarda, Int. J. Mod. Phys. C 16, 515 (2005).

[4] R. Olfati-Saber, Proc. Am. Control Conf. 4, 2371 (2005).

[5] M. Buchanan, G. Caldarelli, and P. De Los Rios, Networks in Cell Biology (Cambridge University Press, Cambridge, 2010).

[6] S. C. Manrubia, A. S. Mikhailov, and D. H. Zannette, Emergence of Dynamical Order (World Scientific, Singapore, 2004).

[7] D. McMillen, N. Kopell, J. Hasty, and J. Collins, Proc. Natl. Acad. Sci. U.S.A. 99, 679 (2002).
[8] T. S. Gardner, D. di Bernardo, D. Lorenz, and J. J. Collins, Science 301, 102 (2003).

[9] F. C. Hoppensteadt and E. M. Izhikevich, Weakly Connected Neural Networks, Applied Mathematical Sciences (Springer, New York, 1997).

[10] B. Bollobás, Modern Graph Theory (Springer, New York, 1998).

[11] T. Biyikoglu, J. Leydold, and P. F. Stadler, Laplacian Eigenvectors of Graphs (Springer, New York, 2007).

[12] A. E. Motter, C. Zhou, and J. Kurths, Phys. Rev. E 71, 016116 (2005)

[13] F. Chung, Ann. Comb. 9, 1 (2005).

[14] A. Motter, C. Zhou, and J. Kurths, Europhys. Lett. 69, 334 (2005). 
[15] R. Agaev and P. Chebotarev, Linear Algebra Appl. 399, 157 (2005).

[16] L. Donetti, F. Neri, and M. A. Muñoz, J. Stat. Mech.-Theory E. (2006) P08007.

[17] A. N. Samukhin, S. N. Dorogovtsev, and J. F. F. Mendes, Phys. Rev. E 77, 036115 (2008).

[18] A. Banerjee and J. Jost, Linear Algebra Appl. 428, 3015 (2008).

[19] P. N. McGraw and M. Menzinger, Phys. Rev. E 77, 031102 (2008).

[20] D. Watts and S. Strogatz, Nature 393, 440 (1998).

[21] A. Barrat and M. Weigt, Eur. Phys. J. B 13, 547 (2000).

[22] M. E. J. Newman, C. Moore, and D. J. Watts, Phys. Rev. Lett. 84, 3201 (2000).

[23] R. Monasson, Eur. Phys. J. B 12, 555 (1999).

[24] J. Jost and M. P. Joy, Phys. Rev. E 65, 016201 (2001).

[25] M. Barahona and L. M. Pecora, Phys. Rev. Lett. 89, 054101 (2002).

[26] F. Mori and T. Odagaki, J. Phys. Soc. Jpn. 73, 3294 (2004).

[27] R. Kühn and J. van Mourik, J. Phys. A 44, 165205 (2011).

[28] C. Grabow, S. Grosskinsky, and M. Timme, Phys. Rev. Lett. 108, 218701 (2012).

[29] A. Arenas, A. Diaz-Guilera, J. Kurths, Y. Moreno, and C. Zhou, Phys. Rep. 469, 93 (2008).

[30] L. M. Pecora and T. L. Carroll, Phys. Rev. Lett. 80, 2109 (1998).

[31] B. Kriener, L. Anand, and M. Timme, New J. Phys. 14, 093002 (2012).

[32] Y. Kuramoto, Chemical Oscillations, Waves and Turbulence (Springer, Berlin, 1984).

[33] J. Acebron, L. Bonilla, C. Vicente, F. Ritort, and R. Spigler, Rev. Mod. Phys. 77, 137 (2005).

[34] S. Kaka, M. R. Pufall, W. H. Rippard, T. J. Silva, S. E. Russek, and J. A. Katine, Nature 437, 389 (2005).

[35] M. Timme, Phys. Rev. Lett. 98, 224101 (2007).

[36] C. Grabow, S. M. Hill, S. Grosskinsky, and M. Timme, Europhys. Lett. 90, 48002 (2010).

[37] D. Witthaut and M. Timme, Phys. Rev. E 90, 032917 (2014).

[38] J. Tegnér, M. K. S. Yeung, J. Hasty, and J. J. Collins, Proc. Natl. Acad. Sci. USA 100, 5944 (2003).

[39] M. Timme and J. Casadiego, J. Phys. A: Math. Theor. 47, 343001 (2014).
[40] M. Rohden, A. Sorge, M. Timme, and D. Witthaut, Phys. Rev. Lett. 109, 064101 (2012).

[41] D. Manik, D. Witthaut, B. Schäfer, M. Matthiae, A. Sorge, M. Rohden, E. Katifori, and M. Timme, Eur. Phys. J.: Special Topics 223, 2527 (2014).

[42] R. Olfati-Saber, J. A. Fax, and R. M. Murray, Proc. IEEE 95, 215 (2007).

[43] M. Timme, F. Wolf, and T. Geisel, Phys. Rev. Lett. 92, 074101 (2004).

[44] M. Timme, T. Geisel, and F. Wolf, Chaos 16, 015108 (2006).

[45] G. Fagiolo, Phys. Rev. E 76, 026107 (2007).

[46] G. Golub and C. Van Loan, Matrix Computations (Johns Hopkins Studies in Mathematical Sciences) (Johns Hopkins University Press, Baltimore, 1996).

[47] P. Lancaster and M. Tismenetsky, The Theory of Matrices, 2nd ed. (Academic Press, Orlando, 1985).

[48] R. M. Gray, Commun. Inf. Theory 2, 155 (2005).

[49] C. Grabow, S. Grosskinsky, and M. Timme, Eur. Phys. J. B 84, 613 (2011).

[50] E. P. Wigner, Proc. Cambridge Philos. Soc. 47, 790 (1951).

[51] C. Porter, ed., Statistical Theory of Spectra: Fluctuations (Academic Press, New York, 1965).

[52] M. Mehta, Random Matrices (Academic Press, New York, 1991).

[53] T. Tao and V. Vu, Bull. Amer. Math. Soc. 46, 377 (2009).

[54] A. Edelman and N. R. Rao, Acta Numerica 14, 233 (2005).

[55] J. Feng, V. Jirsa, and M. Ding, Chaos 16, 015109 (2006).

[56] A. Mirlin and Y. Fyodorov, J. Phys. A 24, 2273 (1991).

[57] Y. V. Fyodorov and A. D. Mirlin, Phys. Rev. Lett. 67, 2049 (1991).

[58] G. Semerjian and L. F. Cugliandolo, J. Phys. A 35, 4837 (2002).

[59] A. J. Bray and G. J. Rodgers, Phys. Rev. B 38, 11461 (1988).

[60] G. J. Rodgers and A. J. Bray, Phys. Rev. B 37, 3557 (1988).

[61] G. J. Rodgers, K. Austin, B. Kahng, and D. Kim, J. Phys. A 38, 9431 (2005).

[62] F. Götze and A. Tikhomirov, Ann. Probab. 38, 1444 (2010).

[63] V. Girko, Theory Probab. Appl. 29, 694 (1985).

[64] H. J. Sommers, A. Crisanti, H. Sompolinsky, and Y. Stein, Phys. Rev. Lett. 60, 1895 (1988).

[65] I. J. Farkas, I. Derényi, A.-L. Barabási, and T. Vicsek, Phys. Rev. E 64, 026704 (2001).

[66] O. Sporns and E. Bullmore, Nat. Rev. Neurosci. 10, 186 (2009). 